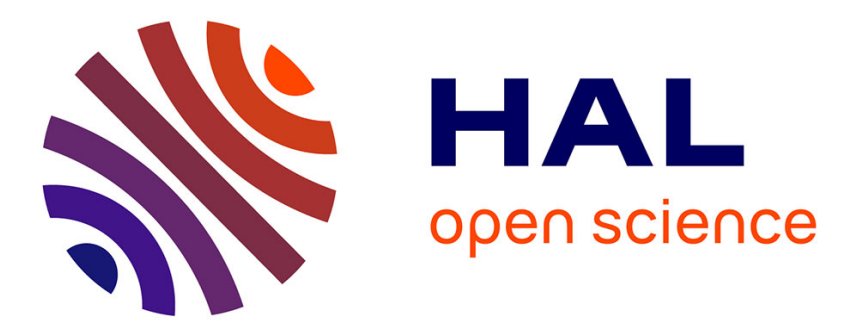

\title{
Acoustic Emission: a new in-line and non-intrusive sensor for monitoring batch solution crystallization operations
}

Gilles Févotte, Nesrine Gherras

\section{- To cite this version:}

Gilles Févotte, Nesrine Gherras. Acoustic Emission: a new in-line and non-intrusive sensor for monitoring batch solution crystallization operations. ADCHEM 2012, Jul 2012, Singapour, France. Paper WeBT2.6 pp.178-185. hal-00731844

\section{HAL Id: hal-00731844 \\ https://hal.science/hal-00731844}

Submitted on 13 Sep 2012

HAL is a multi-disciplinary open access archive for the deposit and dissemination of scientific research documents, whether they are published or not. The documents may come from teaching and research institutions in France or abroad, or from public or private research centers.
L'archive ouverte pluridisciplinaire HAL, est destinée au dépôt et à la diffusion de documents scientifiques de niveau recherche, publiés ou non, émanant des établissements d'enseignement et de recherche français ou étrangers, des laboratoires publics ou privés. 


\title{
Acoustic Emission: a new in-line and non-intrusive sensor for monitoring batch solution crystallization operations
}

\author{
Gilles Févotte \& Nesrine Gherras
}

\author{
Ecole des Mines de Saint Etienne, centre SPIN, LPMG, FRE CNRS 3312. 158, cours Fauriel. 42000 Saint Etienne (France) \\ \& Université de Lyon, Université Lyon 1, 43 bld du 11 Novembre 1918. 69100 Villeurbanne (France) \\ (e-mail: fevotte@emse.fr)
}

\begin{abstract}
Acoustic emission (AE) is shown to provide complex and in-depth information on both the liquid and the dispersed phases during batch cooling solution crystallization processes. Despite its complexity, such information might be highly valuable for process monitoring and control purposes owing to its non-intrusive features, its relative cheapness, and the very wide scope of its potential applications. Basic crystallization phenomena such as the onset of nucleation and the development of crystal growth, several key-process variables like the concentration of solid in suspension, and overall data describing the average particle size and the content in impurities of the crystallization medium are evaluated from real experimental data obtained at the lab-scale. AE is not claimed here to allow replacing "usual" particle sizes sensing technologies like image analysis or FBRM; it is rather suggested that the large amount of information contained in the acoustic data is quite interesting and deserves further investigation.
\end{abstract}

Keywords: Chemical industry, Sensors, Monitoring, Particle size, Crystallization, Batch processing, Calibration, Acoustic emission.

\section{INTRODUCTION}

The pharmaceutical industry is more and more set against strong requests on behalf of both consumers and regulatory agencies to improve the quality of its products as well as its manufacturing practices (The latter are currently referred to as Good Manufacturing Practices, cGMPs). Improving the monitoring and control of industrial crystallizers is therefore a major current industrial concern as it is clearly in strong connection with the need for mastering the properties of solid Active Pharmaceutical Ingredients (APIs.) (Sistare et al., 2005; Yu et al., 2004; Wu \& Khan, 2010)

Crystallization of APIs is widely used as a separation and purification process, and also as a means of generating particles with specified end-use properties. The latter are connected to the drug end-use properties, such as the Crystal Size Distribution (CSD), crystal habit, chemical purity... and are known to exert a significant impact on the therapeutic efficacy of the APIs.

During crystallization many concomitant, changeable and competitive phenomena take place such as primary and secondary nucleation, crystal growth, attrition and/or agglomeration, which are likely to occur in a poorly reproducible way and can result in undesirable batch-to-batch variability of the product.

One of the means of improving the quality of pharmaceutical products is to apply process monitoring and control strategies which, obviously, rely on the development of efficient, robust and practical sensors. Among others, this observation led the US Federal Drug Administration (FDA) to promote the PAT initiative (Process Analytical Technology) aimed at encouraging the pharmaceutical industry to collect real time process information, detect and control possible undesirable variations of process operations and branch out new means of analyzing and correcting past operations.

In this context, measuring supersaturation has firstly been a key-issue because supersaturation is the driving force of almost all basic crystallization phenomena. Several multipurpose and reliable methods for performing supersaturation measurement were developed which are mainly based on the use of in situ spectroscopic techniques: Mid- and Near-infrared, Raman, UV-visible, etc. Secondly, measuring the CSD (Crystal Size Distribution) is obviously a major issue which, in contradiction with supersaturation measurements, has not received flexible, reliable and "routine" conclusive answers. In addition to "standard" particle sizing technologies (e.g. like laser diffraction techniques, coulter counters) which all raise significant difficulties for in-line use, video imaging and processing techniques are today the object of intense research efforts. However, until today, the algorithms proposed in the literature lack of generality, remain complex and computationally demanding and cannot provide quantitative data in dense suspensions. Anyway, it is not the purpose of this paper to compare image analysis with $\mathrm{AE}$, which is still almost unexplored in the field of crystallization control and remains quite an immature technology for industrial use. The goal here is rather to present some partial and preliminary analysis of features of the complex information emitted during crystallization phenomena. 
Acoustic emission is a non-destructive sensing method that has been used for real time process monitoring applications, especially in the field of material testing (Steel industry, aeronautics, automotive industry, material sciences, building industry, etc). Even though applications of AE were less frequently reported in the case of pharmaceutical processes, successful application of AE to monitoring various chemical engineering processes was reported in the past (Birch et al., 2005; Boyd and Varley, 2001). These applications include e.g. fluidized bed granulation, fluidized bed coating, powder compaction, etc (Wadley and Mehrabian, 1984; Sawada et al. 1985; Serris et al., 2002). Among applications to particulate systems however, only few studies deal with the AE monitoring of crystallization processes (Sawada et al., 1985; Ersen et al., 2006; Lube and Zlatkin, 1989). It is the goal of the present study to evaluate potential applications of AE to the monitoring of crystallization.

\section{MATERIAL AND METHOD}

\subsection{Experimental set-up}

The crystallization of Ammonium Oxalate monohydrate (AO in the following) in water was selected as a model solute/solvent system. A schematic of the crystallizer is shown in Fig.1. The initial temperature was about $47^{\circ} \mathrm{C}$ and the initial solute concentration was $0.1 \mathrm{~kg} / \mathrm{kg}$ solution, the solution was thus undersaturated in order to ensure complete dissolution of the solute, as one can see in Fig. 2.

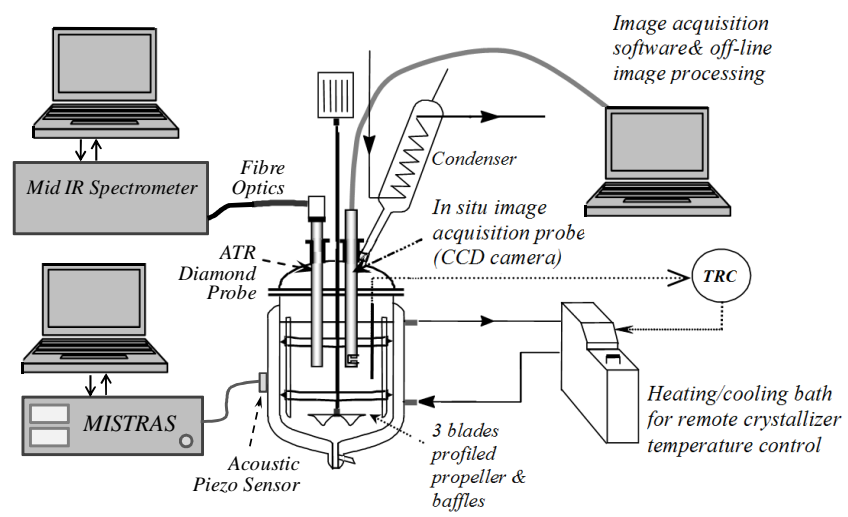

Fig. 1. Schematic of the crystallization setup equipped with ATR-FTIR, in situ image acquisition and AE testing devices.

The experiments were carried out with various cooling rates until the final temperature of $10^{\circ} \mathrm{C}$. As shown in Fig.2, the nucleation burst occurred during the batch unseeded experiments at a temperature depending on the cooling rate. Various cooling rates were applied between $\mathrm{R}=\mathrm{dT} / \mathrm{dt}=-2 \quad \&$ $30^{\circ} \mathrm{C} / \mathrm{h}$ : the temperature of the reactor was controlled so as to track linear temperature trajectories of the reactor content.

\subsection{Instrumentation and experimental procedure}

The ATR FTIR technology (Attenuated Total Reflectance Fourier Transform InfraRed) was used for measuring supersaturation during the experiments (see e.g. Togkalidou et al. 2002). Since the early pioneering works many successful applications of ATR spectroscopy to crystallization control were reported in the open literature. As reported elsewhere (Gherras \& Fevotte, 2012), chemometrics (PLS: Partial Least Squares) allowed obtaining a satisfactory calibration model yielding reliable and accurate measurements of the solute concentration $\mathrm{C}(\mathrm{t})$ despite the significant temperature variations applied during the process. It was thus possible to relate the evolutions of acoustic signals to the development of the process.

An additional in situ imaging probe developed in the University of Lyon (France) was also set in the crystallizer. The probe allowed monitoring the dispersed crystal phase, on the one hand, and estimating the CSD off-line, on the other hand (Ahmad et al., 2011)

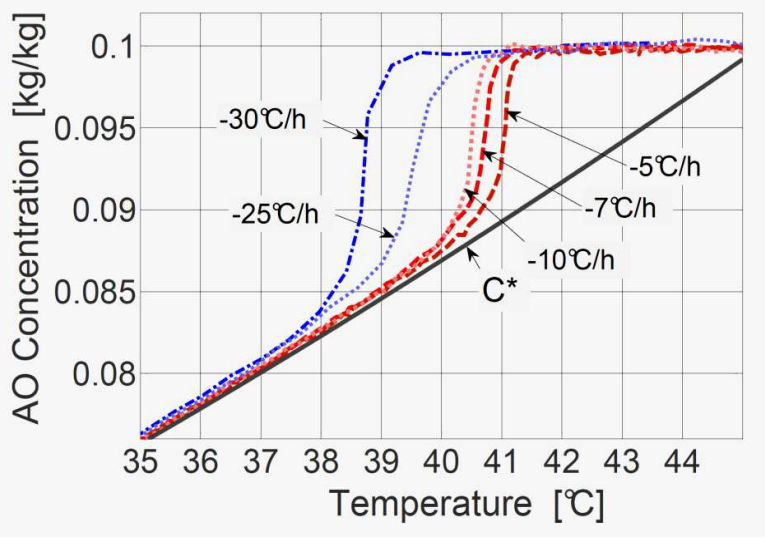

Fig. 2. Concentration profiles during the cooling crystallization of AO in pure water, measured using ATR in situ spectroscopy.

\subsection{The technique of acoustic Emission (AE)}

The measurement is fully passive as it consists in "earing" the noise naturally emitted by on-going physical processes. The AE equipment is therefore based on a transducer acoustically coupled to materials undergoing dynamic changes. The sensor detects the elastic energy of acoustic waves propagating from the physical source of $\mathrm{AE}$ and thus yields information about the dynamic changes taking place in the AE sources (Grosse \& Ohtsu, 2008). Thanks to its nonintrusive and non-destructive features, and to the possibility of developing multipurpose in-line applications, it is shown below that $\mathrm{AE}$ might be a very attractive technique for monitoring batch cooling solution crystallization operations. The objective of this work is thus to demonstrate the potential for using $\mathrm{AE}$ in monitoring batch crystallization processes.

A basic concept behind $\mathrm{AE}$ crystallization monitoring is that phase transitions occurring during crystallization in solution could induce physicochemical changes in the suspension yielding release of energy. Acoustic elastic waves would be thus generated and propagate in the liquid medium. Meanwhile, as crystal particles are generated, the elastic properties of the dispersed phase change. Such changes of suspension properties affect the acoustic emission caused, for example, by the particle collision impacts and inter-particles and/or particles-wall frictions. The elasticity of crystals and their kinetic energy are also affected by many other properties such as size, shape, hardness, density, uniformity 
of composition which can vary according to the many process operating conditions.

The AE equipment consists of a piezoelectric sensor fixed on the wall of the crystallizer. Coupling grease is used to improve the transmission of acoustic signals between the jacket wall and the sensor. Acoustic emission waves produced by crystallization events, such as nucleation or crystal growth, are transmitted from the physical source of emission, via the solution and the crystallizer wall to the sensor where they are converted into an electronic signal. The signal is then conditioned, amplified, filtered and processed by a specific Data Acquisition System from EuroPhysical Acoustics S.A connected to the probe.

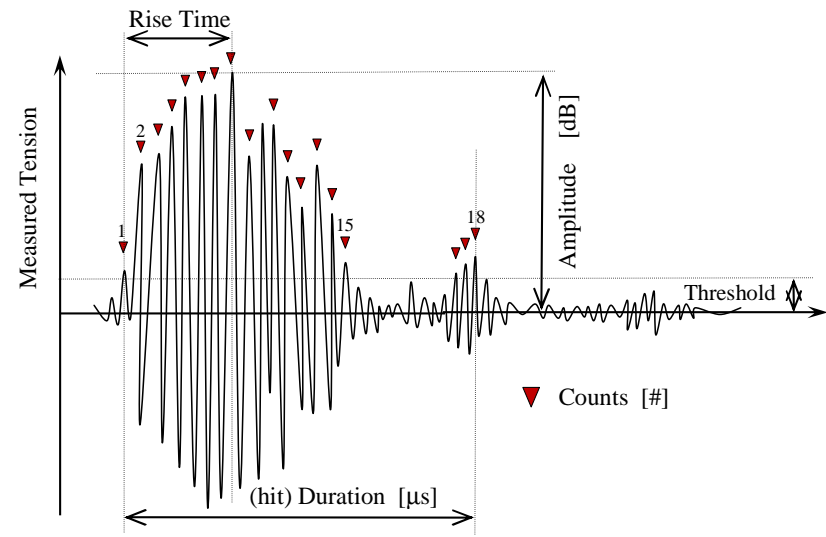

Fig. 3. Main characteristic parameters of a typical AE hit.

A basic parameter used to characterize the level of acoustic signals in the time domain is the root mean square value (RMS) defined as follows where $p(t)$ is pressure magnitude of a continuous acoustic wave taken between times $t_{1}$ and $t_{2}$ :

$$
\tilde{p}_{R M S}=\frac{1}{t_{2}-t_{1}} \int_{t_{1}}^{t_{2}} p(t)^{2} d t
$$

From a practical point of view, as displayed in Fig.3, $p(t)$ is evaluated as an electrical voltage $u(t)$ emitted by the acoustic transducer and amplified by the sensing device. The amplitude is defined as the maximal voltage of the AE signal divided by the reference voltage of the sensor equal to $1 \mu \mathrm{V}$. So we have: $A_{d B}=20 \log \left(u_{\max } / u_{r e f}\right)$.

The frequency $f$ is the average ratio between the number of counts $\mathrm{n}_{\text {counts }}$ and the duration of the burst: $f=\mathrm{n}_{\text {counts }} / \mathrm{d}$.

The peak frequency, in $\mathrm{kHz}$, is the point in the power spectrum at which the peak magnitude is observed, the frequency centroid is the barycentre computed from the sum of the frequencies balanced by the amplitude of the wave, it is thus equivalent to a first moment of inertia. The absolute energy, expressed in atto-Joule $\left(1 \mathrm{aJ}=10^{-18} \mathrm{~J}\right)$ is defined from the integration of the output voltage $u(t)$ of the transducer.

\section{CRYSTALLIZATION OF AMMONIUM OXALATE, PRELIMINARY EXPERIMENTAL OBSERVATIONS.}

3.1 Energy of the acoustic signal of crystallization.
Figure 4 shows the simultaneous time-evolutions of the acoustic signal represented by the measured absolute energy and of the relative supersaturation computed from ATR FTIR data. Supersaturation is expressed as the ratio $\beta=C / C^{*}$ where $\mathrm{C}^{*}$ is the temperature dependent solubility concentration. The cooling rate, $\mathrm{dT} / \mathrm{dt}=30^{\circ} \mathrm{C} . \mathrm{h}-1$, was kept constant during the batch experiment presented in Fig.4.

The development of the batch run in Fig. 4 can roughly be divided into five parts denoted by letters A to E. During Phase A discrete-time early acoustic emission events were recorded. Such detection is quite remarkable because, to the best of our knowledge, no sensor has already been reported to detect such kind of crystallization precursor events. The early detection suggests that $\mathrm{AE}$ might be related to very basic nucleation phenomena.

A clear "primary nucleation peak" takes place during Phase B. Again, the related insert in Fig. 4 shows that much before reaching the so-called limit of metastable zone, the nucleation rate is not nil, which is quite consistent with usual nucleation models. Phase $\mathrm{C}$ follows the decrease of concentration usually associated with the general notion of "primary nucleation burst" (i.e., attainment of some maximal supersaturation value associated with the maximal rate of nucleation). During Phase $\mathrm{C}$, the acoustic emission appears as a continuous signal releasing increasing energy levels. The same signal increases exponentially during Phase D until a relatively narrow peak is reached. During the final Phase E, the energy released decreases continuously and stabilizes at an almost constant level.

In order to get an additional point of view about the crystallization process, the suspension was also monitored using in situ video imaging. The images taken during Phase A show that though they are rare, AO crystals are already present in suspension. The pictures highlight the onset of the intensification of both nucleation and growth processes during Phase A, and also suggest that the AE energy could be related to more and bigger crystals in suspension.

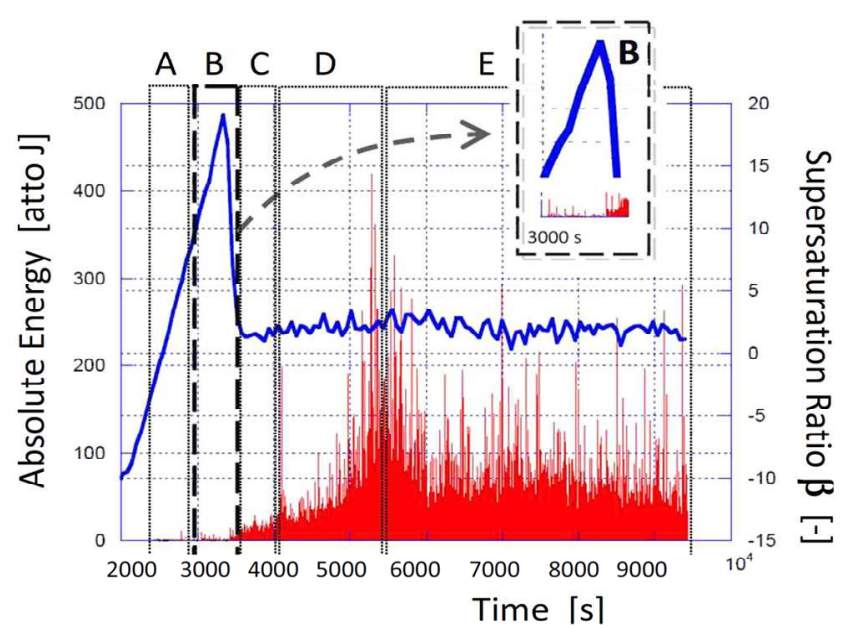

Fig. 4. Relative supersaturation ratio $\beta=C / C^{*}$ and absolute energy measured during the cooling crystallization of ammonium oxalate at a cooling rate of $30^{\circ} \mathrm{C} \cdot \mathrm{h}^{-1}$ 
It seems patent, from the previous observations, that the intensity of $\mathrm{AE}$ is related to the development of the crystallization phenomena (i.e. crystal nucleation and/or growth). However, it turns out to be difficult to identify a clear possibility of discriminating between the contributions of basic phenomena governing the batch process as a whole. For example, it was outlined above that despite similar supersaturation levels, Phases $\mathrm{D}$ and $\mathrm{E}$ exhibit major variations of the energy released through AE. To explain such variations one could reasonably assume that, for similar supersaturation values, differences in the way of converting the dissolved solute into solid phase might result in variations of AE energy. It is likely for example that during phase E, primary nucleation is replaced by secondary nucleation as the prevailing mechanism. However, such basic considerations about the relationship between the level of supersaturation and the recorded absolute energy cannot explain the peak of energy observed at time $5500 \mathrm{~s}$. This simple observation suggests that the solid generation process is not the only source AE.

\subsection{Is crystallization the source of acoustic emission?}

During crystallization, many potential sources might cause the phenomenon of acoustic emission. In order to determine if the development of crystallization generates an acoustic signal apart from any other phenomenon (i.e. stirring, interparticle shocks or shocks of particles against impellers or reactor wall), the cooling crystallization procedure was performed without stirring. AE was found to take place simultaneously with the development of crystallization even though, without stirring, the "acoustic activity" is much less intense. The recorded AE activity is also consistent with the observation of delayed onset of primary nucleation: under stirring, the first occurrence of $\mathrm{AE}$ is recorded after $2888 \mathrm{~s}$ while without stirring the first $\mathrm{AE}$ event is recorded after 5000s. This very basic experiment does not prove that the generation of crystal structure is the only source of AE (i.e., under stirring, one may argue that a major part of the emission are due to stirring effect, particles/impeller shocks, in particular), but it demonstrates that some basic crystallization processes have a noticeable acoustic signature. The latter could therefore be related to crystallization phenomena and used for monitoring crystallization processes.

3.3 Evolutions of the AE frequency variables during batch crystallization

Typical AE parameters recorded during 5 batch cooling experiments performed at 4 constant set-point cooling rates of $-30,-25,-20,-12{ }^{\circ} \mathrm{C} / \mathrm{h}$ were compared. Two kinds of AE variables were basically found to convey information about the development of crystallization processes:

- Firstly, some frequency variables related the AE bursts do not seem to depend on the operating conditions and, therefore, suggest that such kind of information is related to the dispersed solid features (may be size and shape?) and to the crystal structure itself which of course does not depend on the operating conditions. Figure 5e shows that 3 main peak frequencies are observed during the batch cooling operations performed in pure solvent. The recorded hits are reproducible whether one considers the 3 main experimental average frequencies or their order and frequency of appearance. Moreover, the order and the frequency of appearance is ordered in the same way, whatever the experiment. In other words, the bands which are referred below to as $\pi_{1}$ to $\pi_{3}$ in Fig.5e appear successively during the batch time and the number of hits characterized by a given frequency $\pi_{\mathrm{i}}$ are increasing when index $\mathrm{i}$ increases.

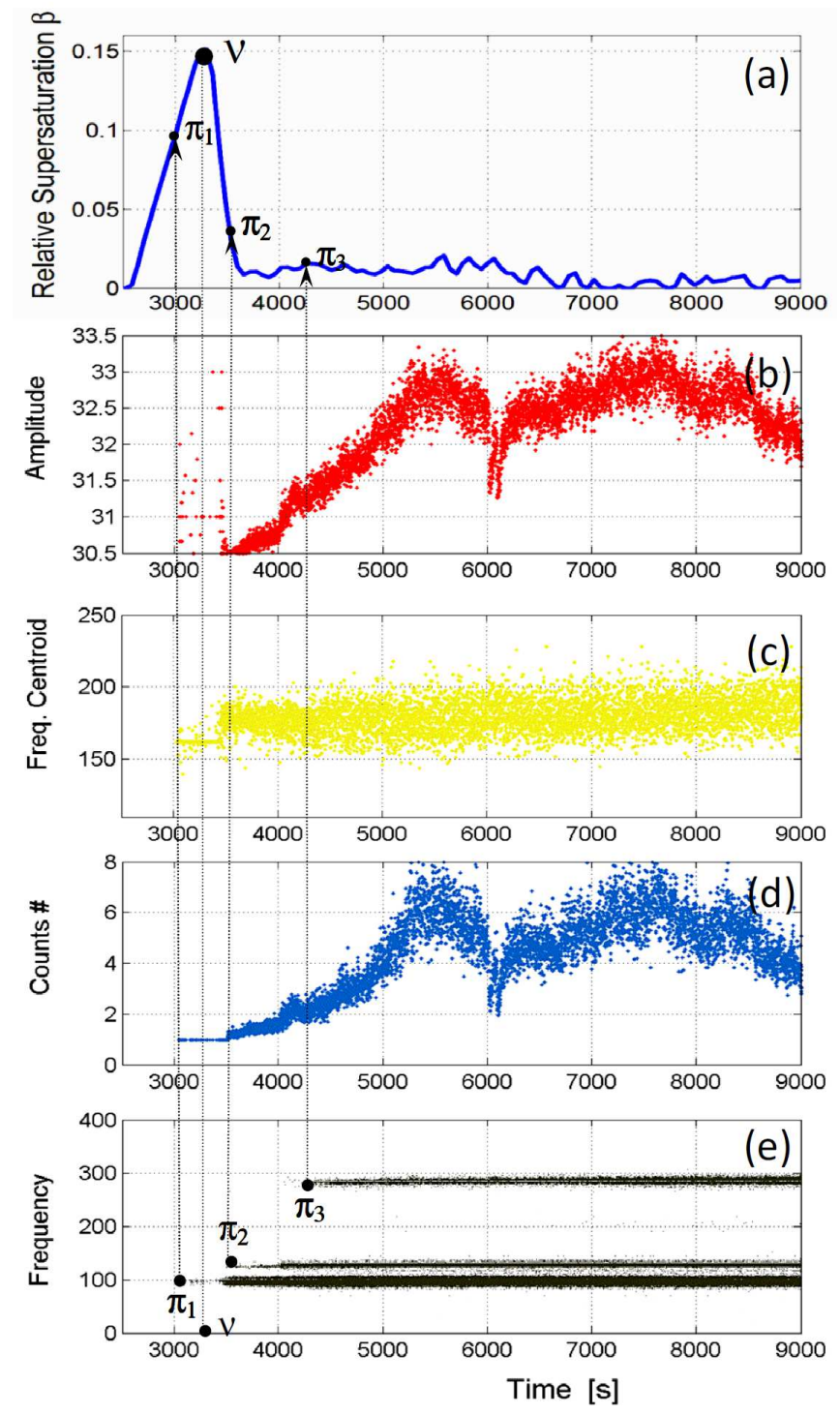

Fig.5. EA characteristic variables: frequency and frequency centroid $(\mathrm{kHz})$, amplitude and number of counts as compared with supersaturation time variations. All variables are computed as moving averages on $10 \mathrm{~s}$ time intervals.

As shown in Fig.5, The AE emission starts at time $\pi_{1}=3040 \mathrm{~s}$ while the limit of metastable zone is reached at time $\pi_{2}=3360 \mathrm{~s}$. Between times $\pi_{1}$ and $\pi_{2}=3510 \mathrm{~s}$ the AE hits are characterized by single peaks. Time $\pi_{2}$ is characterized by the onset of hits exhibiting multiple peaks. It should be noticed that around $\mathrm{t}=5500 \mathrm{~s}$, the maximum number of counts reaches 170 , even though the average never exceeds 8 counts/hit. Every stage denoted by $\pi_{1}$ to $\pi_{3}$ is thus characterized by the appearance of a new frequency lasting until the end of the 
batch experiments: times $\pi_{1}$ to $\pi_{3}$ are related to the appearance of frequencies $f=100,125$ and $285 \mathrm{kHz}$, respectively. Unfortunately, as far as times $\pi_{1}$ to $\pi_{3}$ are set in contrast with the time variations of supersaturation (Fig. 5a), it remains difficult to give any clear explanation of the appearance of new frequencies during the batch cooling process, in connection with basic crystallization phenomena.

- Secondly, the AE variables corresponding to the energy, such as the averaged values of the amplitude, RMS and number of counts appear to depend on the development of the crystallization process itself and, therefore, might by used for monitoring purposes.

3.4 Some observations about quantitative parameters related to the energy of AE.

A detailed modelling work was previously reported showing that, in fact, the crystallization of ammonium oxalate in water is characterized by two different nucleation and growth regimes occurring for $\mathrm{dT} / \mathrm{dt} \in\left[-5,-10^{\circ} \mathrm{C} / \mathrm{h}\right]$ and $\mathrm{dT} / \mathrm{dt} \in[-20$,$30^{\circ} \mathrm{C} / \mathrm{h}$ ] (Gherras \& Fevotte, 2012). Besides these two regimes, the crystallization observed for intermediate cooling rates (in particular for $\mathrm{dT} / \mathrm{dt}=-12^{\circ} \mathrm{C} / \mathrm{h}$ ) was found to be quite erratic and irreproducible. It is interesting to note that for intermediate regimes, the $\mathrm{AE}$ variables were also found to be erratic and irreproducible so that one may also assume that the features of $\mathrm{AE}$ in this latter case could express the observed intermediate crystallization regime.

Fig. 6 displays the results of the dynamic simulation of some characteristic crystallization variables. The simulation is based on the measurements of supersaturation and uses a Population Balance Equation (PBE) model presented elsewhere (Gherras \& Fevotte, 2012). As already outlined, the maximum of energy released (around time 5500s in Fig.6a) does not correspond to the maximum rate of solid production (time 3500s in Fig. 6b). One can also note that $\pi_{2}$ corresponds to a discontinuity of the solid generation process: at time $\pi_{2} \cong 3500$ s the first derivative $\mathrm{dC}_{\mathrm{s}} / \mathrm{dt}$ presents a rather sharp peak related to a significant decrease of the solid production measured thanks to ATR FTIR spectroscopy. The next part present some results aimed at evaluating more precisely the relationships between the formation of solid (i.e. expressed as $\mathrm{C}_{\mathrm{S}}(\mathrm{t})$ ) and $\mathrm{AE}$ energy.

\section{ENERGY OF ACOUSTIC EMISSION AND BASIC CRYSTALLIZATION PHENOMENA}

\subsection{Measurement of solute and solid concentration: Preliminary results}

The dependence of $\mathrm{AE}$ features on the development of crystallization mechanisms can be more thoroughly analyzed thanks to the examination of the many parameters describing the acoustic waves (e.g., number or amplitude of hits, frequency, duration, etc.) as a function of the time variations of the basic mechanisms occurring during the cooling process (i.e. primary and secondary nucleation, crystal growth, etc.)
As already mentioned, the available ATR FTIR solute concentration measurements $\mathrm{C}(\mathrm{t})$ allow computing the time variations of the crystallized solid $\mathrm{C}_{\mathrm{S}}(\mathrm{t})$. Such variations are a good quantitative representation of the crystallization advancement:

$$
\mathrm{C}_{\mathrm{S}}(\mathrm{t})=\mathrm{C}_{\mathrm{o}}-\mathrm{C}(\mathrm{t})
$$

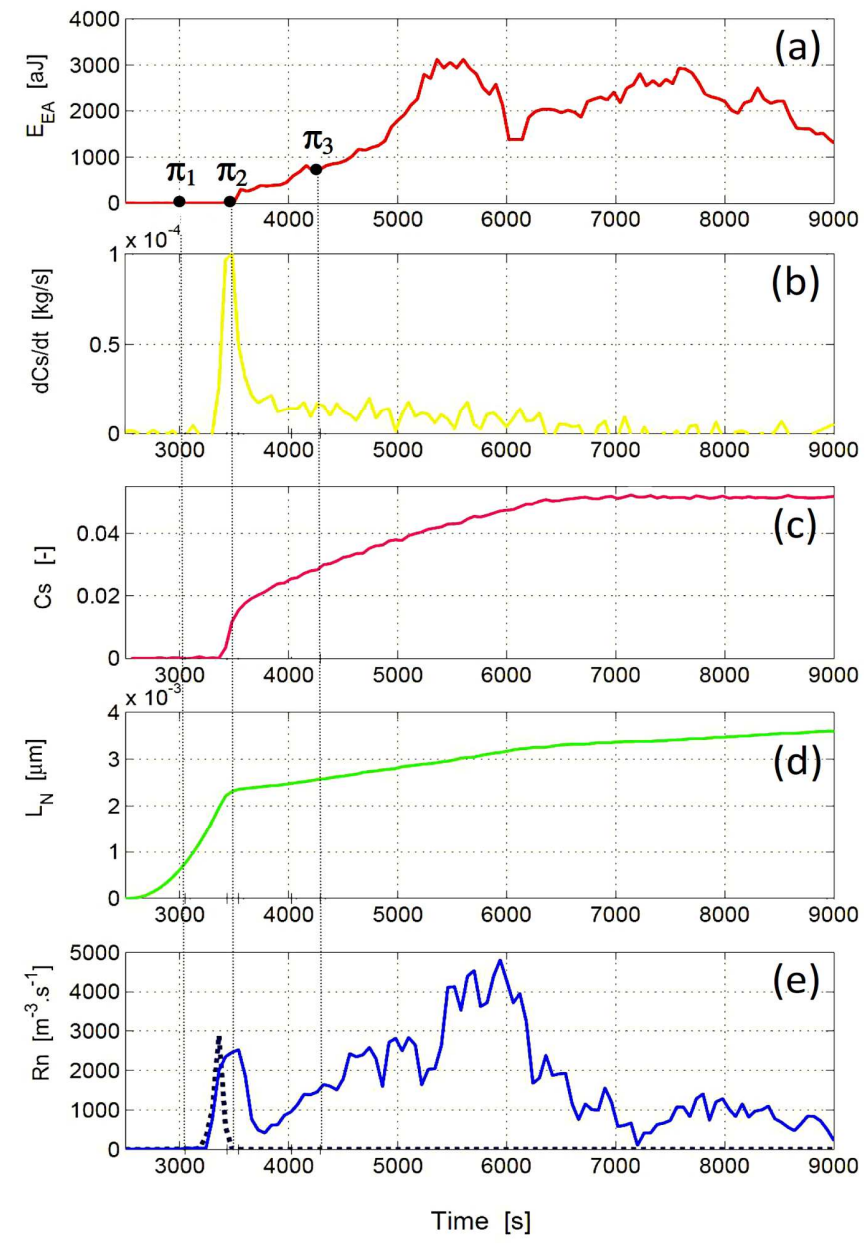

Fig.6. Dynamic PBE simulation of kinetic variables related to the development of the batch process presented in Fig.3. $\mathrm{L}_{\mathrm{N}}$ is the number average particle size and $\mathrm{Rn}$ is the nucleation rate $($ dotted $=$ primary and solid $=$ secondary nucleation rates $)$

Actually, the indirect measurement of $\mathrm{C}_{\mathrm{S}}(\mathrm{t})$ provides a worthwhile means of confronting the development of $\mathrm{AE}$ signals with crystallization. Figure 7 displays the time variations of the cumulative number of counts and absolute energy as a function of the solid concentration. Typical time variations of $\mathrm{C}_{\mathrm{S}}$ are also given in Fig. 7a. The shape of the trajectories shown by most measured $\mathrm{AE}$ variables is similar from one experiment to another, but he range of variation of changes significantly from batch to batch: this is clearly due to the way in which the piezo-sensor is stuck on the crystallizer wall (i.e. the sticking pressure, the thickness of the layer of contact grease, etc, have a significant impact on the overall amplitude of signals).

Should the problem of the reproducibility of acoustic signal be solved (e.g. using an immersed piezo-sensor or acoustic waveguide), then one could design a calibration curve 
relating the cumulative number of counts to the solid concentration and, therefore, develop a new means of measuring supersaturation. This assumption is illustrated in Fig. 8 showing that until a concentration of solid of about $0.04 \mathrm{~kg} / \mathrm{kg}$ (i.e. $80 \%$ solute conversion), the concentration of solid - and consequently the supersaturation $\beta$ - can be estimated from the measured cumulative number of counts.

Fig.8 also shows that, consistently with the previous observation about the features of $\mathrm{AE}$ measurement in the intermediate regime, the previous calibration curve is not valid for experiments performed at cooling rates between -10 and $-20^{\circ} \mathrm{C} / \mathrm{h}$. Moreover, it is clear that, according to the operating conditions, the relationships $\mathrm{Cs}=\mathrm{f}\left(\mathrm{n}_{\text {counts }}\right)$ is no more valid when the solid formation exceeds $80 \%$. A possible explanation for this result is given in the next paragraph.

\subsection{Monitoring both the liquid continuous phase and the dispersed phase using $A E$.}

The evolutions of acoustic energy versus time, displayed in Fig. 4, are similar for the various batch operations performed between $-20^{\circ} \mathrm{C} . \mathrm{h}^{-1}$ and $-30^{\circ} \mathrm{C} . \mathrm{h}^{-1}$. A first period of paraboliclike increasing energy terminates with a sharps peak followed by a final period where the level of recorded absolute energy is almost constant. During the last part of the run, the integrated absolute energy vanishes.

Should the observed AE steps be related to crystallization phenomena occurring during the batch operation, then nucleation and growth would be first involved. A period is likely to follow the first "crystallization dominated" step during which growth and inter-particle shocks could then prevail. A last period where only shocks between the many particles present at the end of the batch would remain is finally likely to take place, due to the cancellation of supersaturation. However, the previous assumptions are rather speculative and do not seem to be so easy to bring to light, as there is no clear correspondence between the development of basic crystallization events and the above mentioned AE phases. As displayed in Fig.3, one can recall that there is no clear apparent correspondence between the $\mathrm{AE}$ and the supersaturation profiles.

One can presume of the possible basic physical phenomena generating AE: nucleation events, crystal growth and particles shock caused by stirring are thus clearly likely to lead to AE. The simultaneous contribution of these 3 phenomena might explain the rather non-trivial energy profile presented in Fig. 3 and the difficulty in relating the course of supersaturation to the time evolutions of the recorded $\mathrm{AE}$ profiles. In this spirit, the 3 following equations are assumed to describe the absolute energy $E A$ released from the generation of solid:

$$
\begin{array}{r}
E A_{N}(t)=k_{1} A_{\text {hom }} \exp \left[\frac{-B_{\text {hom }}}{T^{3}(\ln \beta)^{2}}\right]+ \\
k_{2} A_{S N} C_{S}(t)^{j} \sigma(t)^{i} \varepsilon_{a}{ }^{n}
\end{array}
$$

$$
\begin{aligned}
& E A_{G}(t)=k_{3} \frac{d C_{S}}{d t} \\
& E A_{S}(t)=k_{4} N_{\text {part. }}^{0,5} \bar{L}^{2.8} \\
& E A_{\text {Total }}(t)=\sum_{\varphi=\text { nucl., growth, stirring }} E A_{\varphi}(t)
\end{aligned}
$$

where $N_{\text {part }}$ is the particle number, $\bar{L}$ is the average particle size, $A_{\text {hom \& SN }}$ and $B_{\text {hom }}$ are usual nucleation parameters, $\mathrm{i}, \mathrm{k}$, $\mathrm{n}$ are exponents and $\varepsilon_{\mathrm{a}}$ is the stirring power. Indices $\mathrm{N}, \mathrm{G}, \mathrm{S}$ refer to the contribution of nucleation, crystal growth and stirring to the overall energy of the acoustic emission waves.
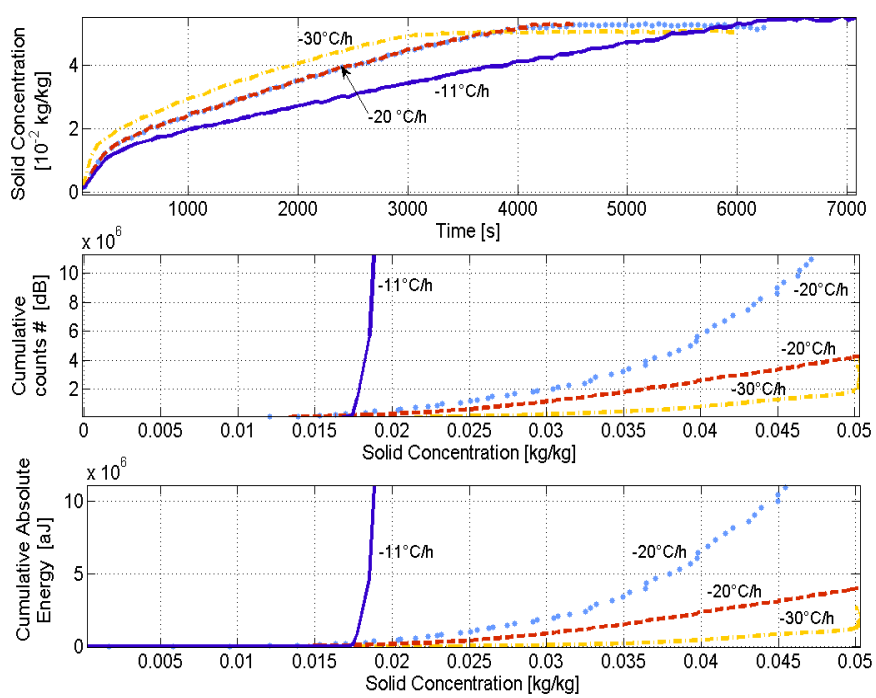

Fig.7. Comparison between the time variations of solid concentration and both the cumulative number of counts and absolute energy during 3 batch cooling operations performed at 3 different cooling rates.

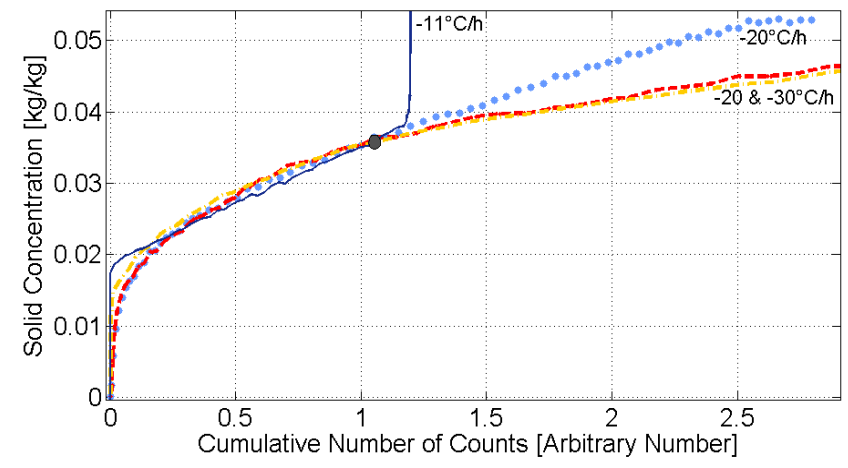

Fig.8. Calibration curve allowing the computation of the solid concentration from the AE cumulative number of counts.

Equation (3) states that the contribution of nucleation to the overall AE energy is proportional to both primary and secondary nucleation rates. The corresponding parameters can be computed from the measured supersaturation variations, as explained in Gherras and Fevotte (2012). The nucleation kinetic parameters involved were reported in the same paper. The second energy component $E A_{G}$ relates the 
generation of solid (i.e., the growth of crystals) to EA absolute energy release and Eq.(5) describes the energy generated by inter-particle shocks, as reported by Guinto et al. (1993). The average particle size was computed from the Population Balance Equation Model (PBE) also presented in Gherras and Fevotte (2012).

Equations (3) to (5), together with the measured values of the degree of supersaturation $\beta(t)$ and the dynamic population model above mentioned allows computing the overall generation of acoustic energy. It was thus possible to minimize a quadratic criteria expressing the error between the measurements displayed in Fig. 3 and the "simulated" values presented in Fig.6.

Such an identification approach was finally successful in determining the four parameters $k_{i}$ leading to the modelpredicted profile displayed in Fig. 9. Despite the relative roughness of the modelling approach, these latter results are quite encouraging as they unexpectedly fit the experimental AE data which, as explained above, appeared to be rather difficult to predict from supersaturation measurements only.

Moreover, as far as the results presented in part 4.1 are concerned, one can now explain why the "calibration curve" presented in Fig. 7 diverges for solid concentrations above $0.4 \mathrm{~kg} / \mathrm{kg}$. Indeed, as the number of particles increases with time, the hypothesis stating that the number of counts and the absolute energy could only be related to the amount of solid present in suspension is less and less valid for increasing solid content. In other word, one can only expect the relationship between the solid concentration and the acoustic absolute energy to be one-to-one when the emission caused by inter-particles shocks and particle/crystallizer wall shocks are negligible.

It is very interesting to note that from the experimental data of $E A_{\text {total }}(t)$ it should be possible to invert (3) to (6) and, $\mathrm{C}_{\mathrm{S}}(\mathrm{t})$ being measured, to compute the average particle size. By doing so, the $\mathrm{AE}$ techniques could allow estimating integral CSD parameters such as average sizes and, therefore, provide highly valuable information on the time variation of the CSD.

In a different way, such kind of measurement was presented recently by Gherras et al. (2011) who presented a calibration of the estimation of the average particle size from EA(t) measurements. The calibration displayed in Fig. 10 was established using experimental $\mathrm{AE}$ data acquired with suspensions containing various solids concentration and particle of different sizes. Even though it cannot be considered as an accurate measurement tool, it is clear that such empirical correlation might be very useful in the industrial practice to estimate and monitor the main trends of the particle generation process (i.e. average particle size and/or overall solid content).

Again, the results presented remain rather speculative and cannot be claimed to allow replacing usual sensing approaches. However, it is our opinion that obtaining even approximate trends of the development of the crystallization process without carrying out complex, costly and time consuming calibration models might be of great interest for process monitoring purposes.

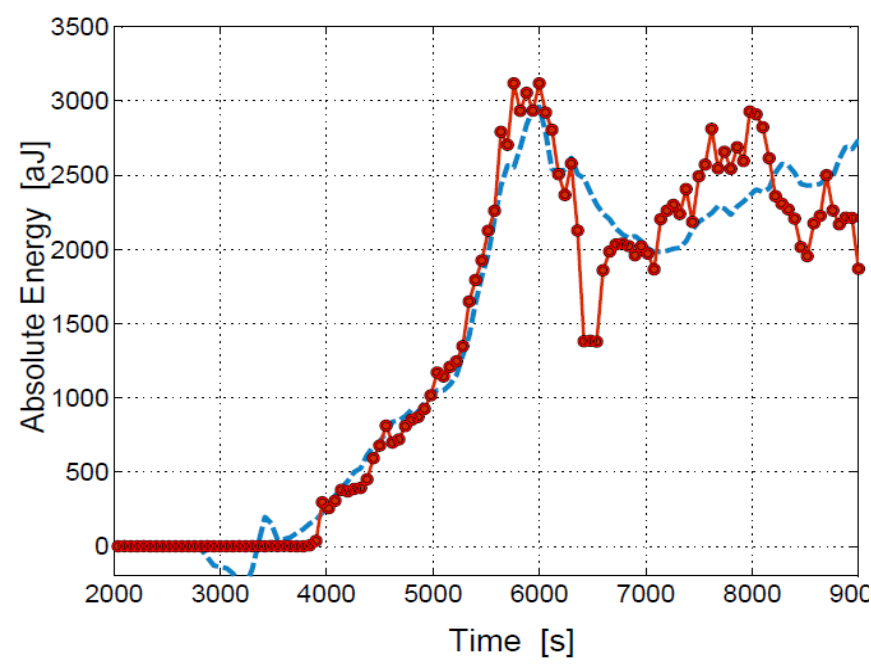

Fig. 9. Fit between the average time variations of the absolute energy recorded during the experiment presented in Fig.3 (dashed line) and the model predictions (dotted line) of the acoustic signal obtained from the least-squares estimation of parameters $\mathrm{k}_{\mathrm{i}}$ in Eqs.(3) to (5).

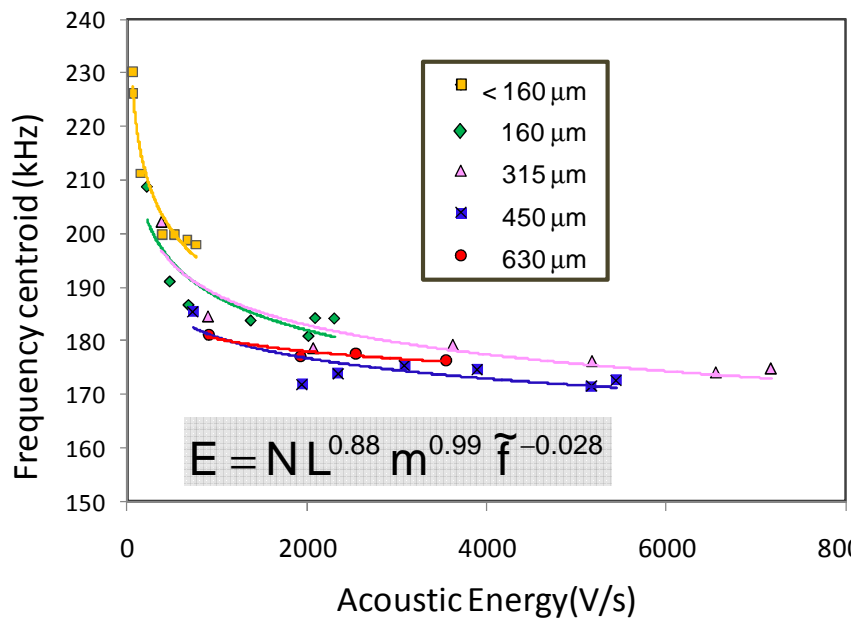

$\mathrm{N}=$ Stirring rate; $\mathrm{L}=$ Average particle size; $\mathrm{m}=$ mass of particles; $\tilde{\mathrm{f}}=$ Frequency centroid

Fig.10. Empirical relationship between the emitted acoustic energy and the frequency centroid as a function of both the total mass of solid in suspension and average particle size.

\section{CONCLUSIONS}

Thanks to the wide scope of information contained in the many recorded acoustic data, acoustic emission might appear as a highly valuable and innovative measurement technique for monitoring solution crystallization processes. The technique is all the more interesting that it is fully non invasive and non destructive, and that acoustic data can be recorded in a very straightforward way, even using portable recording equipments.

Strictly speaking, the preliminary experimental and modelling results presented in this paper are not intended to prove reliable and accurate new applications of the technique to the control of crystallization processes. However, the early 
detection of primary nucleation during cooling solution processes, the on-line estimation of both the solid and the solute concentrations and some rough model-based estimation of the time variations of the average particle size during the crystallization process are suggested to be made possible using in-line $\mathrm{AE}$, without requiring sampling the crystallization medium.

In the actual context of the lack of sensors for monitoring crystallization systems, especially when one deals with the in-line measurement of the CSD, the development of $\mathrm{AE}$ sensing strategies might bring new process information. The latter information deserves to be more deeply investigated and might turn out to be complementary to the actual sensing technologies. At least, one can reasonably think that the preliminary results presented here suggest new qualitative or "semi-quantitative" approaches of monitoring batch crystallization systems. Such monitoring could easily be developed and applied in the industrial context. Among other examples, the early detection of nucleation, the evaluation of detrimental effects of impurities on the development of the crystallization process (not shown here), some approximate monitoring of the solid generation process and the evaluation of rough quantitative parameters describing the CSD (average particle sizes, breakage phenomena, etc) can be identified as valuable possible applications.

\section{REFERENCES}

Ahmad, O.S.; Debayle, J.; Gherras, N; Presles, B; Fevotte, $\mathrm{G}$; Pinoli, JC, 2011. 10th International Conference on Quality Control by Artificial Vision. Bellingham: Spie-Int Soc Optical Engineering.

Birch,M.; Fussell, F.J. ; Higginson, P.D.; McDowall, N. and Marziano, I. 2005. Org.Proc. Res. \& Dev., 9, p.360-364.

Ersen, A., Smith, A. \& Chotard, T., 2006.. Journal of Materials Science, 41(21), p.7210-7217.

Gherras, N. \& Fevotte, G. AIChE J. Available at: http:// onlinelibrary.wiley.com/doi/10.1002/aic.12776/abstract.

Gherras, N. ; Serris E. and Févotte, G. 2011, ISIC $18^{\text {th }}$ Int. Conf. on Indust. Crystallization, ETH, Zurich. http://www.aidic.it/isic18/webpapers/184Gherras.pdf

Grosse, C.U. \& Ohtsu, M., 2008. Acoustic emission testing, Springer.

Guinto, T. Hirajima, M.; Tsunekawa, K.; Tadano, I.; Nakajima, W.; 1993. Adv.Powder Technol., 4(2), p.143-157.

Lube, E.L. \& Zlatkin, A.T., 1989. J. Cryst. growth, 98(4), p.817-826.

Sawada, T. et al., 1985. Anal. Chem., 57(8), p.1743-1745.

Serris, E. et al., 2002. Powder Technol., 128(2-3), p.296-299.

Sistare, F., Berry, L. \& Mojica, C., 2005. Organic Process Research \& Development, 9(3), p.332-336.

Togkalidou, T.; Tung, H.H.; Sun, Y.; Andrews,A.; Braatz, R.D. 2002. Org. Process Res. Dev. 6(3), p.317-322.

W.R. Boyd, J. \& Varley, J., 2001. Chem. Eng.Sci., 56(5), p.1749-1767.

Wadley, H.N.G. \& Mehrabian, R., 1984. Materials Science and Engineering, 65(2), p.245-263.

Wu, H. \& Khan, M.A., 2010. J. Pharmaceutical Sci., 99(3), p.1516-1534.

Yu, L., Lionberger, R.A.; Rawa,A.S.; D'Costa, R.; Wu, H.;
Hussain, A.S. 2004. Adv. Drug Delivery Reviews, 56(3), p.349-369. 